\title{
Neoantigen cancer vaccine augments anti-CTLA-4 efficacy
}

\author{
Erika Salvatori $\mathbb{C}^{1,5}$, Lucia Lione $\mathbb{C}^{1,5}$, Mirco Compagnone $\mathbb{C}^{2}$, Eleonora Pinto $\mathbb{C}^{1}$, Antonella Conforti $\mathbb{C}^{3}$, Gennaro Ciliberto ${ }^{4}$, \\ Luigi Aurisicchio $\mathbb{1}^{1,2,3 凶}$ and Fabio Palombo $\mathbb{1}^{1,2 \bowtie}$
}

Immune checkpoint inhibitors (ICI) based on anti-CTLA-4 (aCTLA-4) and anti-PD1 (aPD1) are being tested in combination with different therapeutic approaches including other immunotherapies such as neoantigen cancer vaccines (NCV). Here we explored, in two cancer murine models, different therapeutic combinations of ICI with personalized DNA vaccines expressing neoantigens and delivered by electroporation (EP). Anti-cancer efficacy was evaluated using vaccines with or without CD4 epitopes. Therapeutic DNA vaccines showed synergistic effects in different therapeutic protocols including established large tumors. Flow cytometry (FC) was utilized to measure CD8, CD4, Treg, and switched B cells as well as neoantigen-specific immune responses, which were also measured by IFN- $\gamma$ ELlspot. Immune responses were augmented in combination with aCTLA4 but not with aPD1 in the MC38 tumor-bearing mice, significantly impacting tumor growth. Similarly, neoantigen-specific T cell immune responses were enhanced in combined treatment with aCTLA-4 in the CT26 tumor model where large tumors regressed in all mice, while monotherapy with aCTLA-4 was less efficacious. In line with previous evidence, we observed an increased switched B cells in the spleen of mice treated with aCTLA-4 alone or in combination with NCV. These results support the use of NCV delivered by DNA-EP with aCTLA-4 and suggest a new combined therapy for clinical testing.

npj Vaccines (2022)7:15; https://doi.org/10.1038/s41541-022-00433-9

\section{INTRODUCTION}

Developing innovative combination protocols to boost the efficacy of immune checkpoint inhibitors (ICl) is one of the challenges in cancer immunotherapy. In the last decade, monoclonal antibodies targeting the CTLA-4 and PD1 pathways have been approved as anticancer drugs for the treatment of several cancer types ${ }^{1}$. More recently it was discovered that the therapeutic success of $\mathrm{ICl}$ is linked to the activation of an immune response against neoantigens ${ }^{2}$, which are tumor-specific mutations recognized by the host immune system ${ }^{3}$. Direct targeting of neoantigens with personalized cancer vaccines is appealing for many reasons, including the potential lack of immunological tolerance and intrinsic tumor specificity. Neoantigen cancer vaccine (NCV) technology has been explored in preclinical tumor models using various delivery systems, including peptides ${ }^{4-10}$, long peptides ${ }^{11}, \mathrm{RNA}^{12,13}$, DNA ${ }^{14,15}$, and nanoparticles ${ }^{16}$. However, promising results in preclinical animal models did not translate in clinical benefit as monotherapy or in combination with anti-PD1 $(\mathrm{aPD} 1)^{17}$ despite the detection of strong immune responses in most NCV-treated patients ${ }^{18-23}$. The lack of correlation requires a more in-depth knowledge of the mechanisms of action of the targeted pathway. Indeed, anti-CTLA-4 (aCTLA-4) and aPD1 are supposed to act at different levels, with the first promoting $T$ cell activation at lymph nodes and the latter reactivating $T$ cells in the tumor $^{24}$. This conclusion is backed up by the large therapeutic advantage of combining aCTLA-4 and aPD1 therapy, even though efficacy comes at the expenses of severe adverse effects ${ }^{25}$. One potential explanation may be the general activation of the immune response against self-antigens expressed by normal tissues, whereas the combination of $\mathrm{ICl}$ with the NCV may spare normal tissue while boosting cancer selectivity.

The efficacy of the ICl-vaccine combination may also be affected by the type of vaccine platform. Previous evidence has shown that vaccine formulation and the vaccination protocol can dictate synergy with aCTLA-4 and aPD1 therapy ${ }^{26}$. The aCTLA-4 role in priming $T$ cell response was investigated with a glioblastoma cell lysate vaccine treated with an NK ligand. A predominant $\mathrm{CD}^{+}$ response and a synergy with $\mathrm{ICl}$ were observed ${ }^{27}$. Moreover, external factors such as the microbiota can modulate the efficacy of a NCV expressed by a plasmid DNA and administered by electroporation $(E P)^{28}$.

Most of the preclinical studies with NCV were conducted with

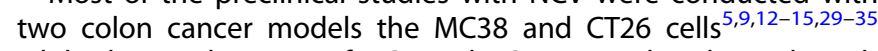
while the combination of $\mathrm{NCV}$ with $\mathrm{ICl}$ was explored mainly with aPD ${ }^{31}$. Here we explored the impact of therapeutic NCV using different vaccination protocols in combination with $\mathrm{ICI}$. Our results suggest that the DNA-EP delivery of NCV is more effective with aCTLA-4 rather than with aPD1 in terms of immunological and antitumor effects.

\section{RESULTS \\ DNA-EP vaccination enhances the antitumor effect of aCTLA-4 in two different colon cancer models}

The amount of immunogenic neoantigens is a vital component in preventing tumor progression, as we and others have established $^{12,15,31}$. To expand on this concept, we generated M8, a DNA plasmid vaccine expressing eight MC38 neoantigens in the form of 28-mers (Table 1). Analysis of the neoantigen individual immune response elicited by $\mathrm{M} 8$ administration in C57BI/6 mice was performed by IFNY ELISpot, confirming vaccine immunogenicity (Fig. 1).

We then asked whether the M8 vaccine could improve the antitumor efficacy when administered in combination with aPD1 and/or aCTLA-4. To this aim, C57BI/6 mice were injected with MC38 cells and M8 administration started two days later in a therapeutic setting. As reported in Fig. 2A, B, the single aCTLA-4 or aPD1 and their combination significantly reduced tumor growth

\footnotetext{
${ }^{1}$ Takis, Rome, Italy. ${ }^{2}$ Neomatrix, Rome, Italy. ${ }^{3}$ Evvivax, Rome, Italy. ${ }^{4}$ Scientific Directorate, IRCCS Regina Elena National Cancer Institute, Rome, Italy. ${ }^{5}$ These authors contributed equally: Erika Salvatori, Lucia Lione. ${ }^{凶}$ email: aurisicchio@takisbiotech.it; palombo@neomatrixbiotech.com
} 
Table 1. M8 vaccine expressing MC38 neoantigens (see Fig. 1).

\begin{tabular}{|c|c|c|c|}
\hline$\#$ & Symbol & WT & Neoantigen \\
\hline 2 & Dpagt1 & SLVISASIIVFNLVELEGDYRDDHIFSL & SLVISASIIVFNLLELEGDYRDDHIFSL \\
\hline 4 & Tmem135 & LLRLTKGRFALMNRKALDVFGTGASREF & LLRLTKGRFALMNLKKALDVFGTGASREF \\
\hline 5 & Spire1 & GEKRSISAIRSYQDVMKICAAHLPTESE & GEKRSISAIRSYQYYVMKICAAHLPTESE \\
\hline 6 & Wbp7 & LSSCLSNFHFMCARASYCIFQDDKKVFC & LSSCLSNFHFMCALASYCIFQDDKKVFC \\
\hline 8 & Nle1 & GHGHWVNTMALSTDYALRTGAFEPAEAT & GHGHWVNTMALSTYYYALRTGAFEPAEAT \\
\hline
\end{tabular}

\section{○ M8 O ELIspot}
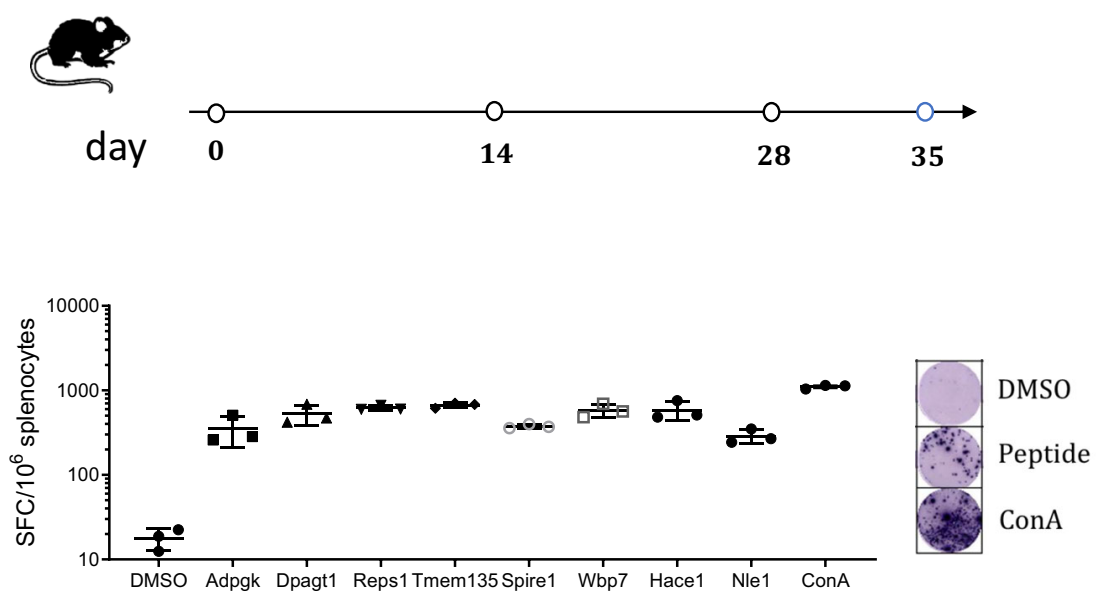

Fig. 1 Immune responses induced by $\mathbf{M 8}$ vaccine. C57BI/6 mice were vaccinated at day 0, 14 and 28, while neoantigen-specific immune responses were analyzed on day 35 on splenocytes by IFN $\gamma$ ELISpot stimulated with individual peptides. ConA served as a positive control. Neoantigen-specific measurements are reported corresponding to the neoantigens listed in Table 1. Each symbol represents an individual sample $(n=3)$. A representative image of the experiment is on the right. Values are from one of two experiments.

while M8 alone did not. Interestingly, the combined therapy of M8 with either aPD1 or aCTLA-4 was not equally effective.

The antitumor effects induced by the M8 vaccine combined with aPD1 were comparable with the aPD1 treatment alone, suggesting that the therapeutic effect was driven by the latter. Similarly, the dual treatment aCTLA-4/aPD1 did not improve over the single therapy. In contrast, the treatment with M8/aCTLA-4 resulted in a significant reduction of tumor growth with respect to aCTLA-4 alone, thus suggesting a possible role of the M8 vaccine in enhancing the antitumoral aCTLA-4 effect. To further support this observation, we evaluated the $T$ cell response induced in the spleen at the time of sacrifices (Fig. 2C). Neoantigen-specific T cell response was significantly increased over the control mice only in mice treated with M8/aCTLA-4, which was even more effective than the triple treatment, M8/aCTLA-4/aPD1, although it did not reach statistical significance. This result suggests a synergistic effect of aCTLA-4 and M8 vaccine delivered by EP.

Recent evidence showed that the presence of a CD4 helper epitope may improve the antitumor response induced by $\mathrm{NCV}^{35}$. To verify this in the context of DNA-EP, we introduced helper CD4 epitopes at the $C$ terminus of the $M 2$ vaccine ${ }^{15}$ containing CD8 epitopes as nonamers, thus generating $\mathrm{M} 2 \mathrm{~h}$ vaccine. FC confirmed the immunogenicity of CD4 epitopes and showed an increase of neoantigen-specific $\mathrm{CD}^{+} \mathrm{IFNY}^{+}$and $\mathrm{CD} 8^{+} \mathrm{TNFa}^{+}$(Supplementary Fig. 1A). This observation was further confirmed by IFNY ${ }^{+}$ELIspot assay (Supplementary Fig. 1B).
Previous evidence showed that CD4 epitopes could be relevant in the cotreatment of CT26 tumors with aCTLA- $4^{36}$. Therefore, to combine CD4 and CD8 neoantigens with $\mathrm{ICl}$ in a second tumor model, we selected CD4 and CD8 neoantigens expressed in the CT26 colon cancer cells ${ }^{37}$ and we generated C20, a vaccine expressing twenty CT26 neoantigens in the form of 28-mers (Supplementary Table 1). In view of the potential role played by the site of vaccination ${ }^{26}$ and the evidence showing effective DNA vaccination with $E P$ in other tissues ${ }^{38}$, we compared the therapeutic effect of C20 vaccine delivered intramuscularly (IM) vs. intradermally (ID). Neoantigen-specific immune response was analyzed by FC one week after last immunization showing significant $\mathrm{CD}^{+} \mathrm{IFNy}^{+}$and $\mathrm{CD}^{+} \mathrm{TNFa}^{+}$responses, which were also significantly elevated as effector memory $\mathrm{CD}^{+} \mathrm{IFN}^{+}$, and $\mathrm{CD}^{+}{ }^{+} \mathrm{TFF}^{+}$in the IM protocol (C20-IM) with respect to control mice (Fig. 3a). Neoantigen-specific $\mathrm{CD}^{+} \mathrm{IFN}^{+}$and $\mathrm{CD}^{+} \mathrm{TNFa}^{+}$ $T$ cells were induced also in C20 delivered ID (C20-ID) but were not statistically significant. To evaluate the immune response against single neoantigens, splenocytes were analyzed. Table 2 summarizes the characterization of the immune responses; we observed two CD8 epitopes (Mtch1, Tmem87), two CD4 epitopes (Aldh1, Dhx35), and two CD8 and CD4 epitopes (E2f8, Slc20a1).

In line with our previous report for the MC38 tumor model ${ }^{15}$, prophylactic C20-IM vaccination resulted in significant tumor delay (Fig. 3B). Reduced tumor growth was also observed in mice vaccinated ID, although it was not statistically significant. To verify 


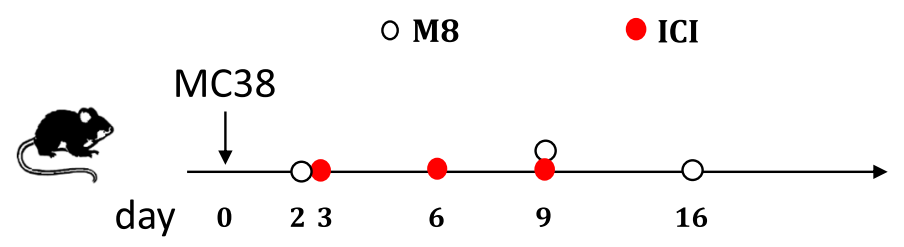

a)

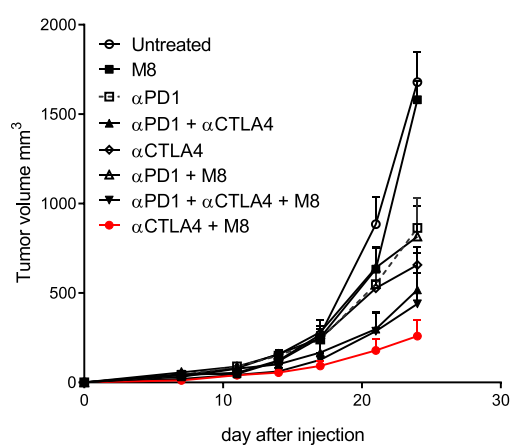

b)

c)
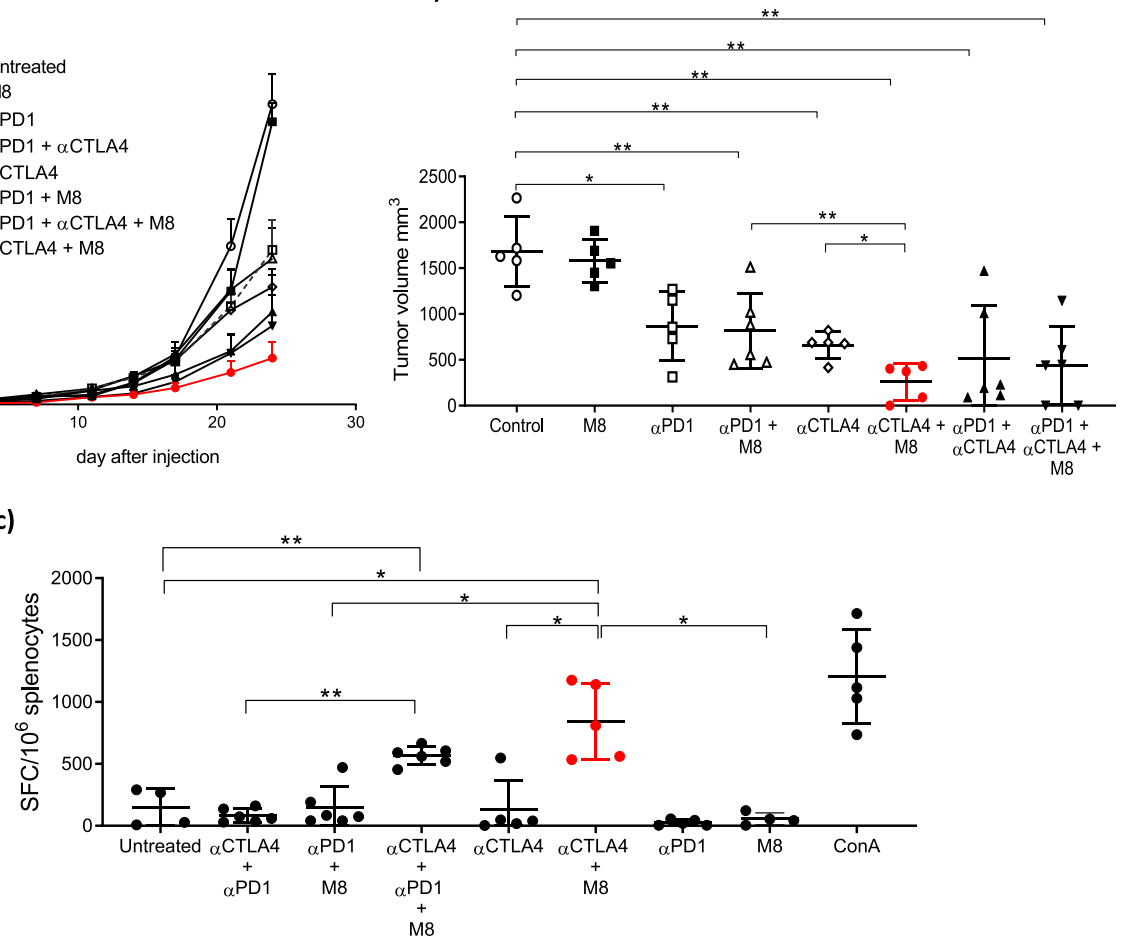

Fig. 2 Therapeutic effect of $\mathbf{M 8}$ in combination with ICl. C57BI/6 mice were inoculated s.c. with MC38 cells and treated with M8 vaccine delivered by EP and $\mathrm{ICl}$ according to the experimental scheme. a Tumor volumes followed over time. $\mathbf{b}$ Final tumor volume measurements, single tumor volumes are depicted from one experiment out of two performed. Mann-Whitney tests were conducted ${ }^{*} p<0,05{ }^{* *} p<0.01{ }^{* * *} p<0.001$. c At day 27, mice were sacrificed and IFN $\gamma$ producing cells were evaluated by IFN $\gamma$ ELISpot assay with splenocytes restimulated with neoantigens pool or ConA as a positive control. Significance was determined using Mann-Whitney test, ${ }^{*} p<0,05{ }^{* *} p<0.01{ }^{* * *} p<0.001$. Six animals per group were utilized. Each symbol represents an individual sample with the error bars representing the s.e.m. Only the most relevant statistics were reported.

whether a different inflammatory response was associated with the reduced antitumor efficacy observed in the C20-ID treated mice, circulating cytokines and chemokines were measured by Luminex multiparameter assay. Out of 14 analytics, only a limited induction of IL17A was observed in mice treated by ID administration (Supplementary Fig. 3A). Finally, we looked at the gene expression using the luciferase reporter gene in the ID vs. IM. A stronger level of luciferase was observed in the IM condition (Supplementary Fig. 2B). Further experiments with more efficient ID protocol and with a larger number of animals are required to explain the inefficient tumor protection in this prophylactic protocol.

Having observed an antitumor effect with the prophylactic C20$I M$ vaccine, we moved to a therapeutic setting also in this tumor model. To better reflect the longer interval used in the clinical schedule, $\mathrm{ICl}$ and the vaccine treatments were performed every five or seven days starting two days after tumor cell implantation. The therapeutic treatment with C20-IM followed by aCTLA4 showed complete tumor regression in cotreated animals, while a partial regression was observed in the group of mice treated only with aCTLA-4 (Fig. 4A). We then investigated the combined treatment in established CT26 tumors with a slightly modified protocol. It has been shown that aCTLA-4 injection the day before a cell-based vaccine induced significant tumor regression of an orthotopic tumor model ${ }^{27}$. Therefore, mice bearing measurable tumors $\left(50-100 \mathrm{~mm}^{3}\right)$ were treated weekly with $\mathrm{ICl}$ and vaccinated the day after with the C20 vaccine for three weeks (Fig. 4B and Supplementary Fig. 4). Tumor stabilization was observed in mice treated with aCTLA-4, resulting in $50 \%$ of survivors at the end of the experiment.

In contrast, tumor regression was observed in $100 \%$ of mice treated with aCTLA-4 and vaccinated with C20-IM (aCTLA-4/C20IM). All mice in this group were tumor-free for more than 250 days. aCTLA-4 followed by C20-ID (aCTLA-4/C20-ID) resulted in a transient prolongation of survival, with some animals relapsing later. Notably, the monotherapy with $\mathrm{C} 20$ vaccine delivered either by ID or IM vaccination was ineffective as aPD1 alone or in combination with C20-IM or C20-ID vaccines. The therapeutic efficacy of aCTLA-4/C20-IM was confirmed in a second experiment up to day 70 when mice were challenged on the opposite flank with the CT26 tumor cells (Supplementary Fig. 4A). To verify whether a memory immune response was induced, survival mice were sacrificed at day 258 and memory immune response was analyzed in the splenocytes. A clear signal was observed in mice treated with aCTLA-4/C20-IM (Supplementary Fig. 5). In line with this evidence, mice treated with aCTLA-4/C20-IM were $100 \%$ protected in the rechallenge experiment (Supplementary Fig. 4B). To characterize the mechanism of action of aCTLA-4/C20-IM we 

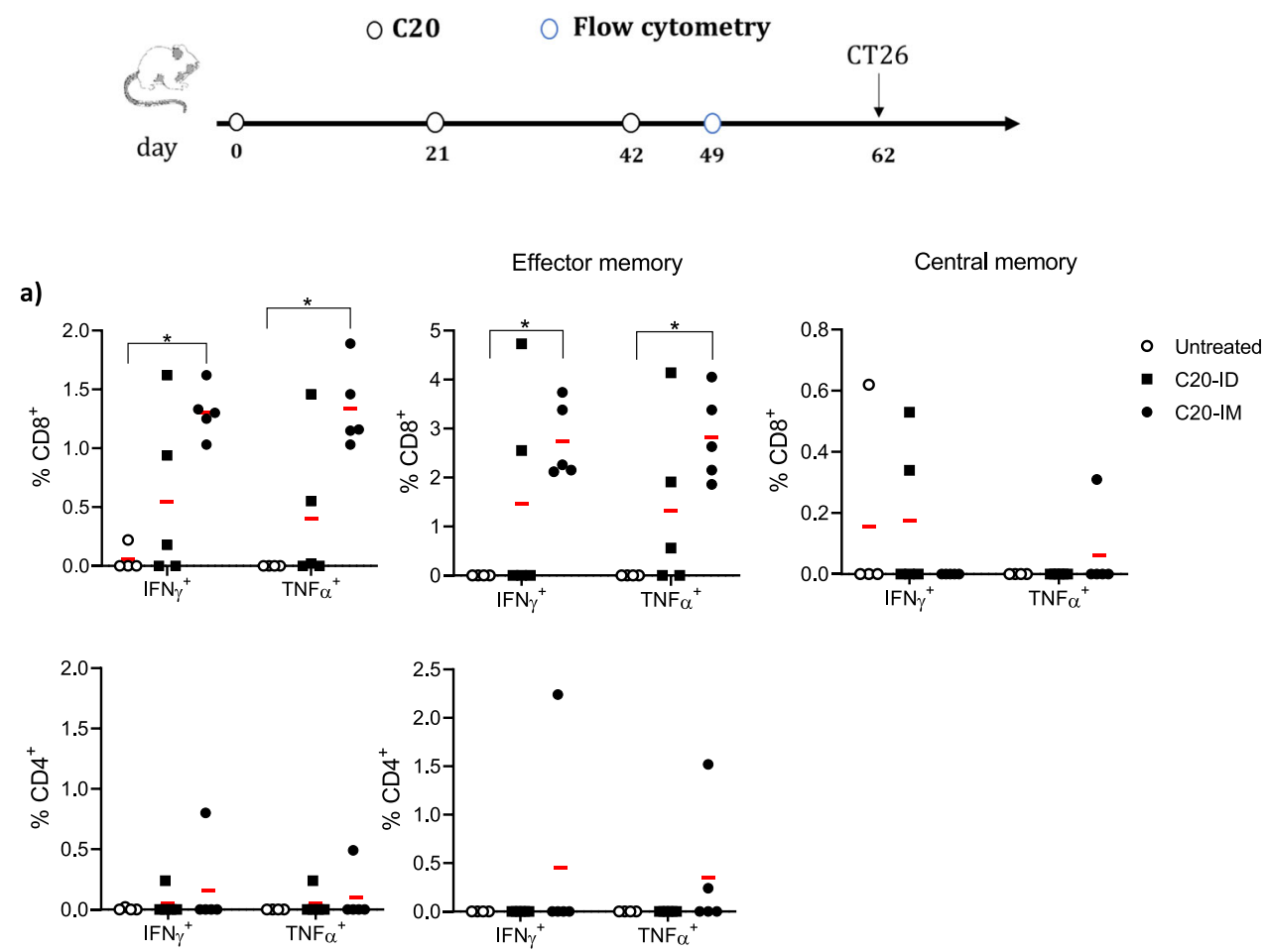

b)

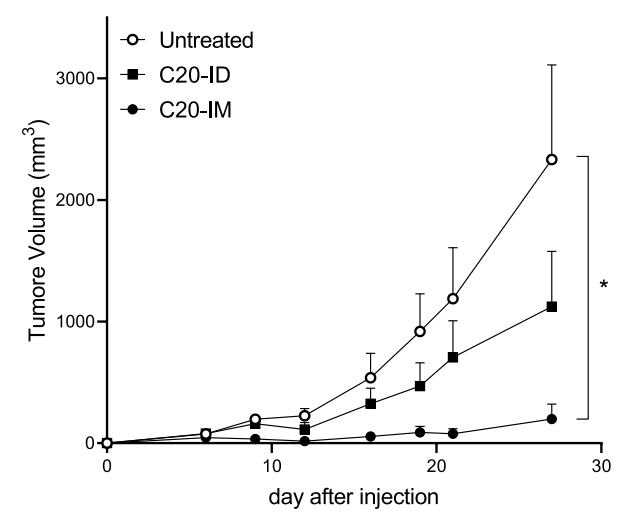

Fig. 3 Prophylactic C20-IM induces a significant reduction in tumor growth. a Balb/c mice were vaccinated with the C20 vaccine at days 0 , 21 , and 42 and challenged with CT26 cells on day 62. One week after the last immunization (day 49), mice were bled retro-orbitally to monitor $\mathrm{T}$ cell immune response against CT26-neoepitopes by intracellular staining. Panel describes CD8 and CD4 neoantigen-specific effector and central memory T cell responses measured by FC using the gating strategy depicted in Supplementary Fig. 2. The stimulation pool included the 15 peptides listed in Table 2. b The panel depicts CT26 tumor growth overtime of one out of two experiments performed. Five animals per group were utilized. Each symbol represents an individual sample with the error bars representing the s.e.m. Significance was determined using Mann-Whitney test $\left({ }^{*} p<0,05\right)$.

performed T cell depletion. Both anti CD4 and anti CD8 abolished anti-tumor activity induced by aCTLA-4/C20-IM (Fig. 4C). These results extend to the $C T 26$ tumor model the combination of NCV delivered by DNA-EP and aCTLA-4 and suggest that the protocol with $\mathrm{ICl}$ followed by NCV could be more effective.

\section{Neoantigen specific $T$ and switched B cells correlate with tumor regression in mice treated with aCTLA-4 and C20-IM}

To identify immune correlates with the antitumor activity observed against established CT26 tumors, neoantigen-specific T cell immune responses were analyzed in peripheral blood by FC (Fig. 5). Tumor-bearing mice showed scattered immune responses. Treatment with aCTLA-4/C20-IM showed a significant neoantigenspecific $\mathrm{CD}^{+}{ }^{+} \mathrm{FN} \gamma^{+}$in all mice and $\mathrm{CD}^{+} \mathrm{TNFa}^{+} \mathrm{T}$ cell response in five out of six. C20-IM elicited a neoantigen-specific peripheral $\mathrm{CD}^{+}{ }^{+} \mathrm{IFNy}{ }^{+}$in four out of six mice that were lessened to only one mouse in the cotreatment with aCTLA-4. In contrast, a consistent and significant neoantigen-specific $\mathrm{CD}^{+}{ }^{\mathrm{TNFa}^{+}} \mathrm{T}$ response, although at a low extent, was induced by aCTLA-4 alone in all treated mice. These responses further increased in mice treated with aCTLA-4/C20-IM. Treatment with aCTLA-4/CD20-ID showed a significant increase in $\mathrm{CD}^{+}{ }^{+} \mathrm{TNFa}^{+}$but not in $\mathrm{CD}^{+} \mathrm{IFNy}^{+} \mathrm{T}$ cells. The results suggest that the anti-tumor mechanism mediated by aCTLA-4 treatment in the IM vaccination may skew the neoantigen-specific immune response toward a $\mathrm{CD} 8^{+} \mathrm{IFN} \gamma^{+}$ effector function whereas in the ID vaccination in the direction of $\mathrm{CD}^{+} \mathrm{TNFa}^{+}$. Treatment with aPD1/C20-IM or aPD1/C20-ID resulted in less frequent measurable responses in two or three out of six mice. We then asked whether the neoantigen-specific 


\begin{tabular}{|c|c|c|c|c|c|c|}
\hline Gene & 28mer & 15 mer mutated & WT & $\begin{array}{l}\text { IFN } \gamma \text { ELIspot Assay } \\
\text { (SFC } 1 \times 10^{6} \text { cells) }\end{array}$ & CD4 (\%) & CD8 (\%) \\
\hline \multirow[t]{2}{*}{ Aldh1 8a1 } & HSGQNHLKEMAISVLEARACAAAGQ & QNHLKEMAISVLEAR & QNHLKEMAIPVLEAR & 141 & 0,055 & - \\
\hline & & KEMAISIVLEARACAA & KEMAIPVLEARACAA & 124,5 & 0,08 & - \\
\hline \multirow[t]{3}{*}{ E2f8 } & ILPQAPSGPSYATYLQPAQAQMLTP & LPQAPSGPSYATYLQ & LPQAPSGPSYAIYLQ & 612 & 0,032 & - \\
\hline & & PSGPSYATYLQPAQA & PSGPSYAIYLQPAQA & 594 & - & 0,045 \\
\hline & & SYATYLQPAQAQMLT & SYAIYLQPAQAQMLT & 45,5 & - & 0,44 \\
\hline \multirow[t]{2}{*}{ Mtch1 } & SWIHCWKYLSVQS吕LFRGSSLLFRR & HCWKYLSVQSSOLLFR & HCWKYLSVQSGQLFR & 822 & - & 0,24 \\
\hline & & YLSVQSSQQLFRGSSL & YLSVQSGQLFRGSSL & 158 & not tested & not tested \\
\hline \multirow[t]{2}{*}{ Tmem87 } & QAIVRGCSMPGPWRSGRLLVSRRWSVE & GCSMPGPW & GCSMPGPWGSGRLLV & 733,5 & - & 0,58 \\
\hline & & PGPWRSSGRLLVSRRW & PGPWGSGRLLVSRRW & 457 & not tested & not tested \\
\hline Agxt2/2 & EHIHRAGGLFVADAIQVGFGRIGKHFW & HIHRAGGLFVADAIQ & HIHRAGGLFVADEIQ & 47,5 & - & - \\
\hline
\end{tabular}

immune responses were measurable in survival mice at day 258. Mice cotreated with aCTLA-4/C20-IM showed a neoantigenspecific polyfunctional $\mathrm{CD} 8^{+} \mathrm{IFN}-\gamma^{+} \mathrm{TNFa}^{+} \mathrm{CD} 8$ effector memory $T$ cell response which was even more evident for the CD4 cells with respect to the other mice (Supplementary Fig. 6). It is tempting to speculate that the long-lasting effector memory response can contribute to the survival observed in mice treated with a aCTLA-4/C20-IM.

We then looked at the intratumoral lymphocytes as compared to splenocytes. $\mathrm{CD}^{+}$and $\mathrm{CD}^{+} \mathrm{T}$ cells showed an increased frequency in the tumor but not in the spleen while nonsignificant trend was observed for $\mathrm{CD} 19^{+}$B cells (Supplementary Fig. 7A). In contrast, a statistically significant increase of neoantigen-specific $\mathrm{T}$ cells was detected in both tissues of C20-IM vaccinated mice (Fig. 6A). Analysis of granzyme b expression revealed a nonsignificant trend (Supplementary Fig. 7B). CTLA-4 is expressed by regulatory $T$ cells and it has been shown that in mice but not in humans aCTLA-4 can reduce this cell population ${ }^{39}$. In this experimental setting we did not observe a reduction of the frequency of $\mathrm{CD}^{+}{ }^{+}$Foxp3 $^{+}$Treg cells (Treg) but a significant increase of neoantigen-specific CD8/Treg ratio (Fig. 6B, C). Other factors may contribute to a strong immune response, recent evidence suggests a role of switched B cells in the antitumor activity mediated by aCTLA $-4{ }^{40}$. To check this, we measured the frequency of $\mathrm{CD} 19^{+} \mathrm{IgG}{ }^{+}$cells. A statistically significant increase was observed in the splenocytes of mice treated with aCTLA-4 alone or with C20-IM with respect to control mice or mice vaccinated with C20-IM (Fig. 6D). This effect was specific for aCTLA-4 in the splenocytes and was not observed in mice treated with aPD1. Altogether these results further support the combined treatment of aCTLA-4/ C20-IM. Further experiments are required to correlate this observation with the enhanced antitumor effect.

\section{DISCUSSION}

The creation of a successful therapeutic protocol that combines ICI and NCV is currently a work in progress. The induction of multiepitopic and multi-functional immune responses by NCV was shown to be relevant in the MC38 tumor mode ${ }^{15}$ and other tumor models ${ }^{31,41}$. Here we extended this concept and explored the strength of DNA-EP platform confirming the immunogenicity of neoantigen previously used in other delivery systems or in the form of shorter coding sequencing (Tables 1 and 2). With the goal of improving the therapeutic index of aCTLA-4 and aPD1, we set experimental conditions that resulted in the expected efficacy for $\mathrm{ICl}^{42}$. Unlike other NCV delivery technologies showing synergetic effects with aPD1 $1^{31,43,44}$, NCV delivered by DNA-EP was more effective in conjunction with aCTLA-4. In the MC38 tumor model, the reduced tumor volume correlated with an increased neoantigen-specific CD8 immune response in the splenocytes (Fig. 2C) and the peripheral blood of CT26 tumor model (Fig. 5A). This observation is coherent with the role of CTLA-4 in regulating $T$ cell activation. It has been shown that, upon TCR ligation, CTLA-4 is upregulated and outcompetes CD28 for B7 ligand binding limiting positive costimulation by $\mathrm{CD} 28^{45}$. An additional mechanism of action of aCTLA-4 is the upregulation of CD86 on migratory $\mathrm{DC}^{46}$, which in turn should favor a stronger $T$ cell response. In line with the role played by aCTLA-4, we observed a statistically significant induction of a neoantigen-specific $\mathrm{CD}^{+}{ }^{+} \mathrm{TFa}^{+}$in tumor-bearing mice (Fig. 5D). This low level of induction was not observed in parallel treatment with aPD1 and was significantly boosted by C20IM treatment further supporting the synergistic effect.

We investigated multiple therapeutic protocols and found that aCTLA-4 administration on the day before NCV treatment is the more effective schedule for established tumors. A similar protocol was originally explored in an orthotopic tumor model ${ }^{27}$. The mechanism of action of the cell lysate vaccine was based on the activation of NK and CD4 T cells. Here we applied this schedule with NCV and showed that effector neoantigen-specific immune responses were induced in the periphery as well as in the tumor. Differently from C20-ID, the C20-IM vaccine protocol resulted in a $\mathrm{CD}^{+} \mathrm{IFN}^{+}$effector memory (Fig. 3A and Supplementary Fig. 6A), and in the therapeutic treatment with aCTLA-4/C20-IM resulted in long-lasting regression of the CT26 tumor model (Fig. 5B). Although the neoantigen-specific immune response in the periphery of tumor-bearing mice was not a clear biomarker of the antitumor response it suggests that the quality of the responses rather than the site of vaccination, ID vs. IM, is an important determinant. Our preliminary data identify some differences in the immune responses between C20-ID and C20- 
a)
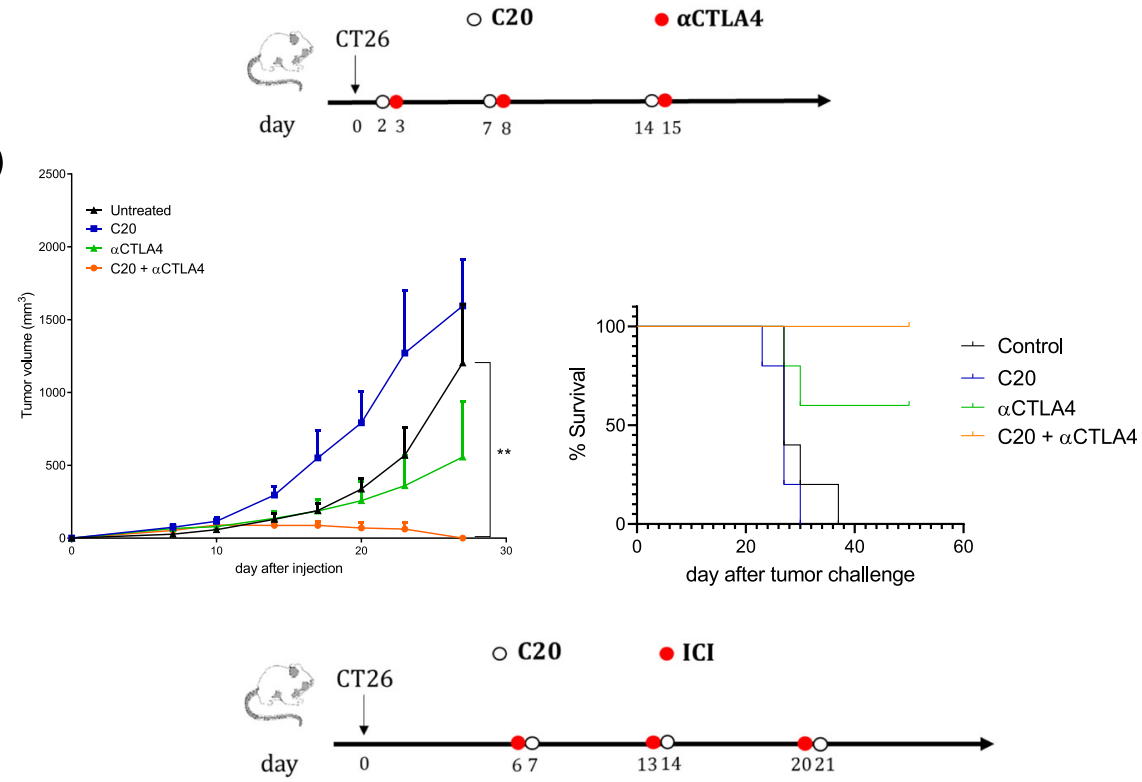

b)
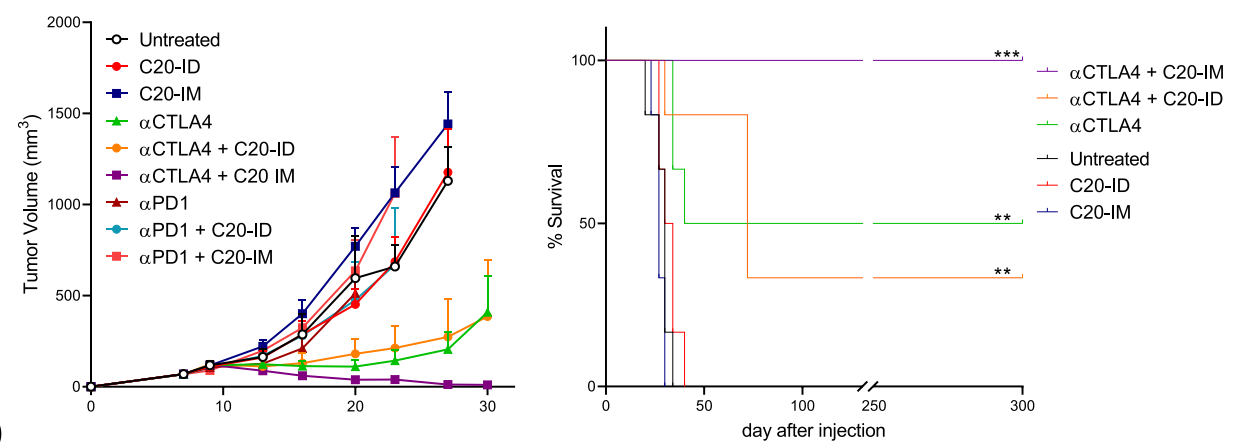

c)

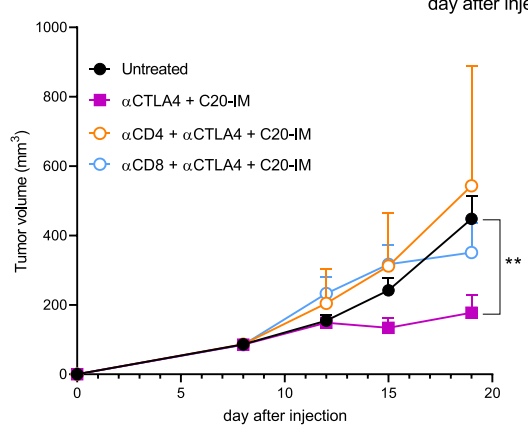

Fig. 4 Therapeutic effect of $\mathbf{C 2 0}$ in combination with ICI in two different schedules of vaccination. a Balb/c mice were inoculated s.c. with CT26 cells and treated with $\mathrm{C} 20$ and $\mathrm{ICl}$ starting from day 2 as depicted in the experimental scheme. Tumor volume and survival curve. b, c Balb/c mice were inoculated s.c. with CT26 cells and treated with ICl and NCV according to the experimental scheme. b Tumor $\left(50-100 \mathrm{~mm}^{3}\right)$ bearing mice were randomized at day 6 and treated with $\alpha C T L A-4$ and vaccinated with C20 the day after. The treatment was repeated weekly as described in the scheme. b Tumor volume measurements and survival curve. This experiment was conducted only once (C) CT26 tumor growth in CD4 or CD8 depleted mice treated as in panel (b). This experiment was repeated twice with similar results. Six animals per group were utilized with the error bars representing the s.e.m. Significance was determined using Mann-Whitney and Log-rank (Mantel-Cox) test ${ }^{* *} p<0.01{ }^{* * *} p<0.001$.

IM, however, we cannot exclude that other electrical conditions may favor neoantigen-specific $\mathrm{CD}^{+} \mathrm{IFN}^{+} \mathrm{T}$ cells immune response in the ID protocol ${ }^{47,48}$. The lack of antitumor activity of NCV with aPD1 in this therapeutic protocol is in line with previous evidence showing that treatment with aPD1 before vaccination had a negative impact on tumor growth ${ }^{49}$. The mechanism seems to be based on the induction of dysfunctional PD $1{ }^{+} \mathrm{CD} 38^{\text {hi }} \mathrm{CD} 8^{+} \mathrm{T}$ cells in sub optimally primed CD8 T cell conditions induced by tumors. Previous evidence in mice and humans showed that nonreversible chromatin modification is induced in non-responding patients and at late-stage tumor growth in preclinical animal models with the CD8 cells expressing $\mathrm{CD} 38$ and $\mathrm{CD} 101^{50}$. The possibility that priming of $\mathrm{T}$ cell response is requested before aPD1 is likely to occur in our experimental conditions. The data from Verma and colleagues $^{49}$ also suggest that in the absence of a good $T$ cell priming, i.e. by NCV, the therapeutic potential of T cells targeting neoantigens may be irreversible committed to a nonresponsive phenotype. 

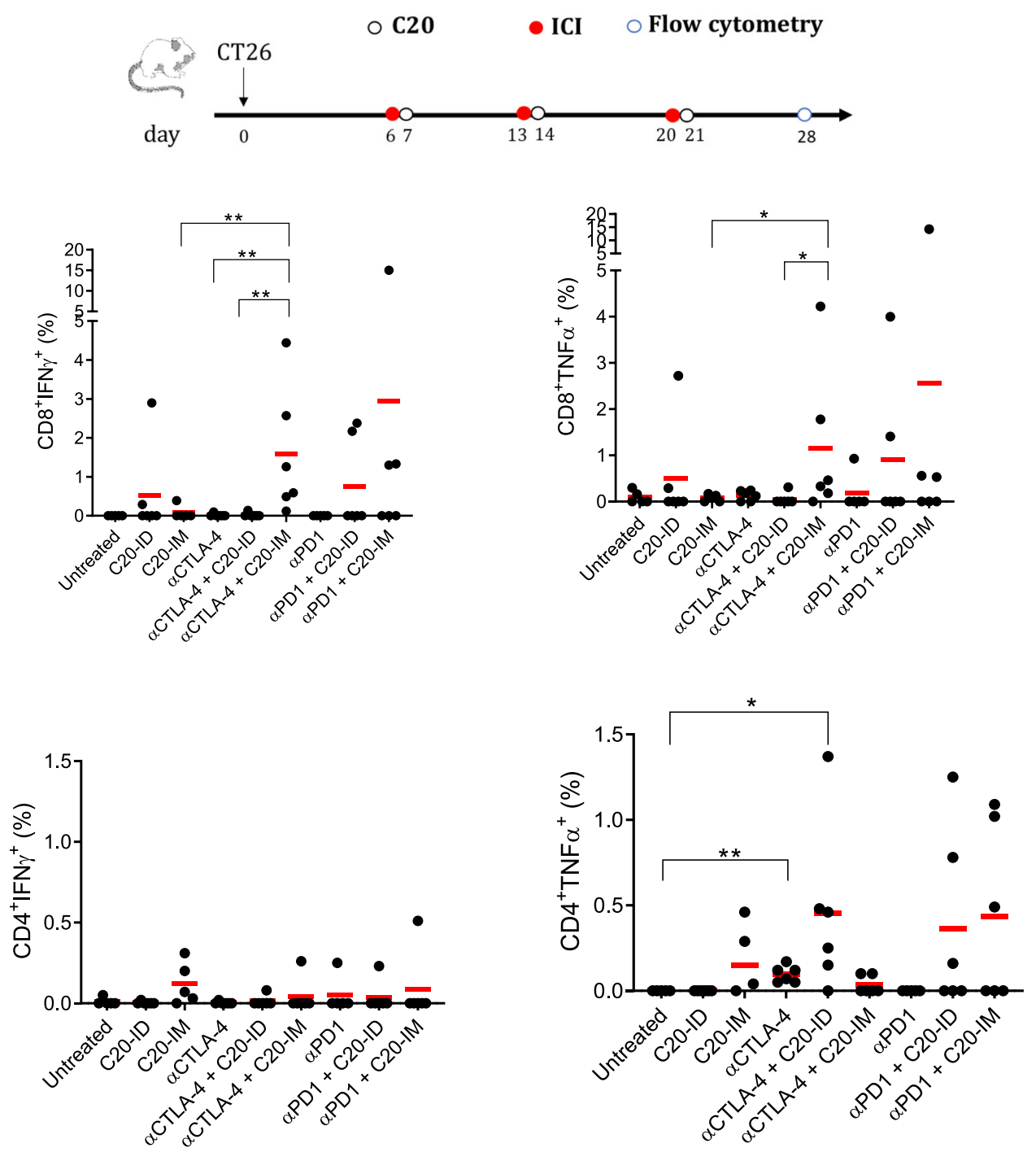

Fig. $5 \mathrm{CD8}^{+} \mathrm{IFNY}^{+}$response increased in aCTLA-4 + C20-IM treated mice. Tumor-bearing mice were treated as described in the experimental scheme and neoantigen-specific immune response was analyzed by FC in the peripheral blood one week after the last treatment (on day 28). Production of IFN $\gamma$ and TNF $\alpha$ by $C D 4^{+}$and $\mathrm{CD} 8^{+} \mathrm{T}$ cells was measured by FC using the gating strategy depicted in Supplementary Fig. 2 upon restimulation with the pool of neoantigen peptides listed in Table 2. This experiment was conducted twice with similar results. Each symbol represents an individual sample $(n=6)$. Significance was determined using Mann-Whitney test, ${ }^{*} p<0,05{ }^{* *} p<$ 0.01 .

The mechanism of action of NCV and aCTLA-4 does not involve only the strengthening of peripheral CD4 and CD8 neoantigenspecific immune response (Fig. 5) but correlates with switched $B$ cells in the spleen (Fig. 6). Importantly, our findings are specific for aCTLA-4. Thibult et al. described aPD1 antibodies directly activating peripheral B cells that had high PD1 expression in a T cell-independent mechanism ${ }^{51}$. In addition, they did not observe any impact of aPD1 on the production of class-switched antibodies. Others have shown that PD1-high B cells functionally suppress $T$ cell activity ${ }^{52}$. In the combined treatment with aCTLA4, we observed a slight but not significant decrease of neoantigenspecific immune responses associated with $100 \%$ protection in the CT26 tumor model. The increased frequency of antibody switched $B$ cells observed in aCTLA- 4 treated mice is in line with previous evidence in other tumors using aCTLA-4 alone or with aPD1. An increased $\mathrm{B}$ cell activation $\left(\mathrm{CD} 19^{+} \mathrm{MHC}-\mathrm{II}^{+} \mathrm{CD} 80 / \mathrm{CD} 86^{+}\right)$, as well as an increased antibody titer against the cancer cells, was observed in breast cancer tumor models ${ }^{40}$. In the CT26 tumor model, we could observe a statistically significant increase of switched B cells only with aCTLA-4 and not with aPD1 in the spleen where they may serve as antigen-presenting cells boosting the $T$ cell response.

The therapeutic protocols utilized were well tolerated including the treatment with dual $\mathrm{ICl}$, which are associated with relevant side-effects in the clinic. Although mouse tumor models are not commonly utilized for toxicology studies, we did not observe any sign of discomfort during the 3 weeks treatment in C57/B6 and Balb/C mice or in the long follow-up in aCTLA-4 and C20 treated mice. It is tempting to translate this in the clinic where a therapy based on DNA-EP and aCTLA-4 could be more appealing than aCTLA-4/ aPD1 due to possible reduced side effects. In conclusion, our data support the use of NCV delivered by DNA-EP with aCTLA4 suggesting new protocols for clinical testing.

\section{METHODS}

\section{Vaccines, cell lines, and mice}

Vaccines specific for each tumor cell line were generated as a fusion protein with the TPA leader sequence upon codon optimization. As for the MC38-specific vaccine, named M8, the neoantigens listed in Table 1 were 

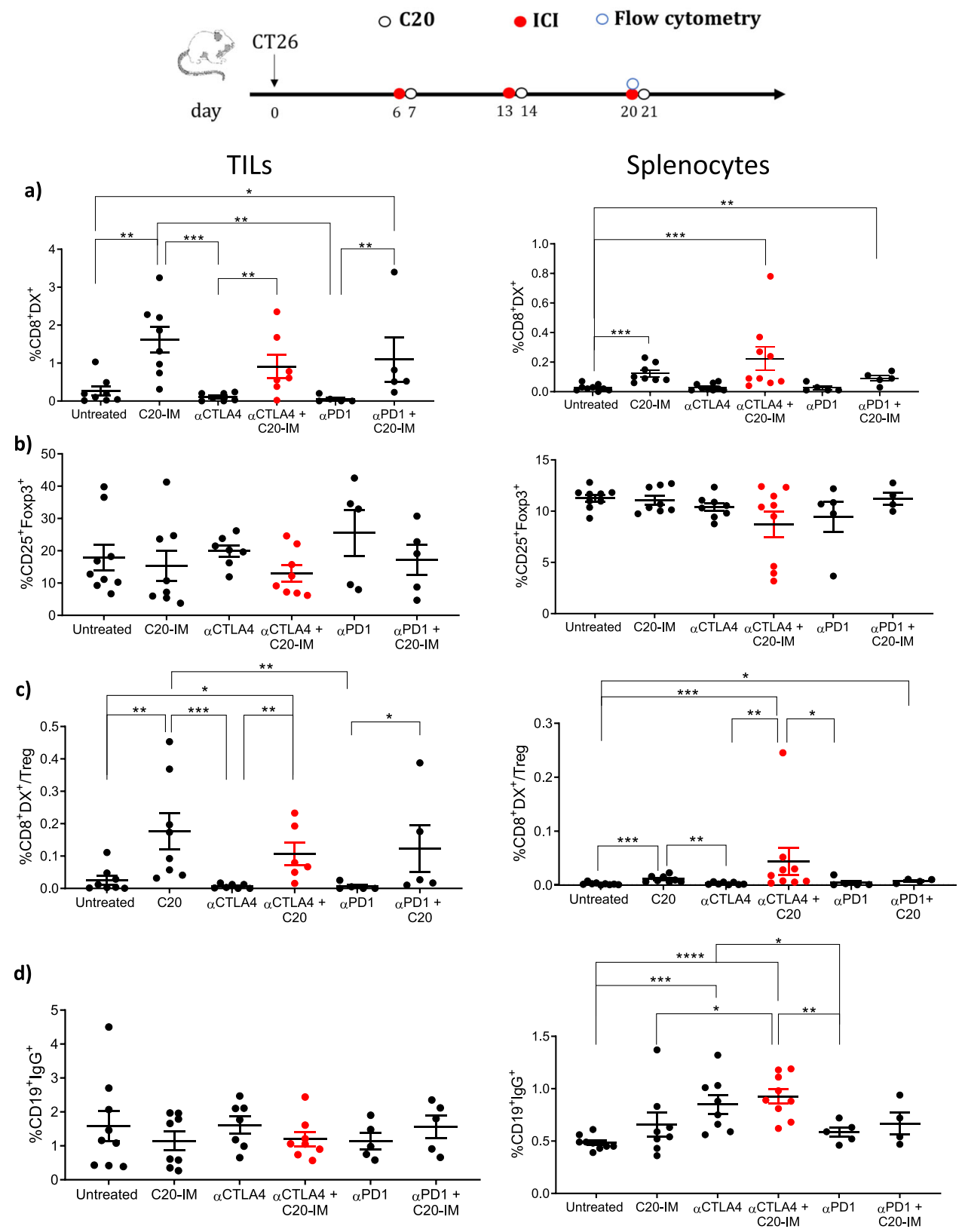

Fig. 6 C20 and aCTLA-4 increased neoantigen-specific CD8 and switched B cells. On day twenty, nine mice per group were sacrificed and tumors and splenocytes were analyzed by FC using the gating strategy depicted in Supplementary Fig. 2. a Frequency of E2f8 neoantigenspecific response was measured using the specific dextramer $(\mathrm{Dx}+)$ gated on $\mathrm{CD} 45^{+} \mathrm{CD} 3^{+} \mathrm{CD} 8^{+}$in live cells. b Treg population $\left(\mathrm{CD} 25^{+} \mathrm{Foxp}^{+}\right)$were gated on $\mathrm{CD} 45^{+} \mathrm{CD}^{+} \mathrm{CD}^{+}$in live cells and expressed as ratio with $\mathrm{DX}^{+}$cells (c). $\mathbf{d}$ Switched $\mathrm{B}$ cells were identified as $\mathrm{CD} 19^{+} \mathrm{IgG}^{+}$cells and gated on $\mathrm{CD} 5^{+} \mathrm{CD}^{+}$in live cells. Nine animals per group were utilized and each symbol represents an individual sample. Significance was determined using Mann-Whitney test ${ }^{*} p<0.05^{* *} p<0.01{ }^{* * *} p<0.001{ }^{* * * *} p<0.0001$.

inserted one after the other without linkers; M2h was produced for the MC38 cell line by inserting at the $C$ terminus of M2 vaccine ${ }^{15}$ the CD4 helper-epitopes FNNFTVSFWLRVPKVSASHLE, AKFVAAWTLKAAAW, and AWLEAQEEEEVGF ${ }^{53}$ were connected one after the other using furin linkers C20 plasmid vaccine specific for the CT26 cells was generated with the neoantigen listed in Supplementary Table 1 without linkers. MC38 and CT26 colon carcinoma cell lines were purchased from Kerafast and ATCC respectively. Master and working cell banks were generated upon receipt, with their third and fourth passages being used for all tumor challenge experiments. Cells were mycoplasma-free as per internal regular controls. In vivo luciferase expression was measured using Xenogen IVIS300 as previously described ${ }^{54}$ Female $6-8$ weeks old C57BL/6 and Balb/c mice (Envigo) were housed in the Plaisant animal house according to national legislation and kept in standard conditions according to Takis ethical committee approval. All the in vivo experimental procedures were approved by the local animal ethics council. The ethical committee of the Italian Ministry of Health approved this research, authorization \# 586/ 2019-PR. Mouse experiments were conducted with a variable number of animals as described in the figure legends.

\section{Immunization schedule}

In total, $10 \mu \mathrm{g}$ of plasmid DNA, were injected in a $50 \mu \mathrm{L}$ volume into the tibialis muscle followed by electroporation, as previously described ${ }^{15}$. For ID administration, C20 were injected in a $30 \mu$ volume intradermally in the left flank and followed by EP (40 V, 3 pulses, $100 \mathrm{~ms}$ length, $120 \mathrm{~ms}$ pause) as previously described ${ }^{55} .200 \mu \mathrm{g}$ of anti-PD1 or anti-CTLA4 were injected in a $200 \mu \mathrm{L}$ volume in mice peritoneum. Tumor challenge was performed by injecting $3 \times 10^{5} \mathrm{MC} 38$ cells on C57BI/6 mice or $1 \times 10^{6} \mathrm{CT} 26$ on Balb/c mice subcutaneously (s.c.), in a $100 \mu \mathrm{L}$ PBS volume in the right flank of the 
mice. Tumor growth was monitored twice a week using an electronic caliper.

\section{In vivo depletion of CD4 and CD8}

CD4 and CD8 were depleted in vivo using anti-CD4 (clone GK1.5, Bioxcell) and anti-CD8 (clone 53-6.7, Bioxcell) antibodies. $200 \mu \mathrm{g}$ of antibody were injected in a $200 \mu \mathrm{L}$ volume in mice peritoneum, twice a week for three weeks.

\section{Immune responses}

The neoantigen-specific immune responses were determined in splenocytes, PBMCs, and tumors of the mice by using intracellular cytokine staining (ICS) performed by flow cytometry (FC), as previously described ${ }^{15}$. Briefly, PBMCs or splenocytes were incubated for $10 \mathrm{~min}$ at room temperature in ACK (Ammonium-Chloride- Potassium) Lysing Buffer (Life Technologies) and then washed in RPMI-1640 medium (Gibco-BRL) supplemented with $10 \%$ fetal bovine serum (FBS, Life Technologies). Tumors harvested from the mice were mechanically dissociated using a scalpel and incubated for $40 \mathrm{~min}$ at $37^{\circ} \mathrm{C}$ with a cocktail of enzymes from the Miltenyi Tumor Dissociation kit in RPMI-1640. Then, tumors were passed through a $70 \mu \mathrm{m}$ Cell Strainer and washed in RPMI-1640 medium with $10 \%$ FBS. For FC analysis of IFN $y$ and TNFa producing cells, $1 \times 10^{6}$ PBMCs or splenocytes were cultured in 96-well plates and stimulated for $12-16 \mathrm{~h}$ in $10 \%$ FBS-supplemented RPMI-1640 with the pool of MC38 or CT26 neoantigen peptides at the final concentration of $5 \mu \mathrm{g} / \mathrm{ml}$, DMSO or PMA-ionomycin, as previously described ${ }^{15}$. For FC analysis, dead cells were excluded by using Fixable Viability Stain 575 V (BD, cat. 565694). Cells were incubated with the anti-Fcy receptor (2.4G2) followed by staining with the following antibodies according to different panels: CD3 (142-2C11) AF488 diluted 1:300 (Thermo Fisher Scientific, cat. 53-0031-82), FOXP3 (FJK-16s) PE diluted 1:100 (Thermo Fisher Scientific, cat. 12-5773-82), CD4 (RM4-5) PerCP-eFluor 710 diluted 1:400 (Thermo Fisher Scientific, cat. 46-0042-82), CD19 (1D3) PC7 diluted 1:300 (Thermo Fisher Scientific, cat. 46-0042-82), CD8 (53-6.7) APC-eFluor 780 diluted 1:300 Thermo Fisher Scientific, cat. 470081-82), CD45 (30-F11) efluor 450 diluted 1:500 (Thermo Fisher Scientific, cat. 48-0451-82), lgG1 (A85-1) BV605 diluted 1:200 (BD, cat. 563285), lgG2a/ b BV605 (BD, cat. 744294), CD25 (PC61.5) SB780 diluted 1:100 (Thermo Fisher Scientific, cat. 78-0251-82), IFNy (XMG1.2) PE diluted 1:150 (Thermo Fisher Scientific, cat. 12-7311-82), TNFa (MP6-XT22) PC7 diluted 1:400 (Thermo Fisher Scientific, cat. 25-7321-82) and MHC Dextramer (H-2Dd/ SGPSYATYL) PE (Immudex) (1:25 dilution) specific for the E2f8 neoantigen. The stained samples were acquired through a CytoFLEX flow cytometer (Beckman Coulter), and the data were analyzed using CytExpert software (Beckman Coulter). Cytokine expression in the presence of only DMSO (with no peptides) was considered background and subtracted from the values measured with stimuli.

\section{IFN- $\boldsymbol{\gamma}$ ELIspot}

The assay was performed on splenocytes from vaccinated and control mice according to the manufacturer's instructions (Mouse IFN-gamma ELISpotBASIC ALP, Mabtech). Briefly, standard 96-well plates (Millipore) were coated with anti-mouse IFN $\gamma$ antibody diluted to $15 \mu \mathrm{g} / \mathrm{ml}$ in sterile PBS and blocked with RPMI- 1640 medium with $10 \%$ FBS. Splenocytes were plated at $4 \times 10^{5}$ and $2 \times 10^{5}$ cells/well, in duplicate, with the pool of MC38 and CT26 neoantigen at the final concentration of $1 \mu \mathrm{g} / \mathrm{ml}$. After overnight stimulation at $37^{\circ} \mathrm{C}$, plates were washed and incubated with biotinylated anti-mouse IFN $\gamma$ antibody, washed, and incubated for $1 \mathrm{~h}$ at room temperature with streptavidin-AP conjugated antibody. After washing, $50 \mu \mathrm{l} /$ well of the substrate (NBT/BCIP-1 step solution, Pierce) was added to measure spot development. After incubation of about $30 \mathrm{~min}$ at RT, the plates were thoroughly washed with distilled water to stop the reaction. Plates were allowed to air-dry completely, and spots were counted using an automated ELISPOT reader (Aelvis ELIspot reader, A.EL.VIS Gmbh).

\section{RT-PCR}

RNA was extracted from $30 \mathrm{mg}$ of frozen tumor tissues according to the manufacturer's instructions (RNeasy kit, QIAGEN). To smash and homogenize tissues, a TissueLyser LT (QIAGEN) was used and operated at $50 \mathrm{~Hz}$ for $2 \mathrm{~min}$. RNA extracted was retro-transcribed to CDNA using a high capacity CDNA Reverse Transcription Kit (Applied Biosystems) according to the manufacturer's instructions. Real-time (RT)-PCR was performed using TaqMan ${ }^{\text {TM }}$ Gene
Expression Master Mix (Applied Biosystem) and commercial TaqMan probes Gzmb genes (Applied Biosystem). Gene expression was normalized to 18S and expressed using the $2^{-\Delta c t}$ method.

\section{Luminex}

Circulating cytokines and chemokines were measured by Luminex multiparameter assay. IL10, IL1b, IL2, IL4 IFNy, TNFa, IL17, IL7, IL6, GMCSF, MIG (CXCL9), IP10 (CXCL10), MIP1a (CCL3), and MCP1 (CCL2) were analyzed according to the manufacturer's instruction.

\section{Statistical analysis}

Log-rank (Mantel-Cox) and Mann-Whitney were utilized where indicated. All analyses were performed in GraphPad Prism 8.0.2.

\section{Reporting summary}

Further information on research design is available in the Nature Research Reporting Summary linked to this article.

\section{DATA AVAILABILITY}

The data not described in the supplementary figures, including raw data and sequences, are available from the corresponding authors upon reasonable request. M8 and C20 plasmid were deposited in Addgene data base (\#80536).

Received: 15 August 2021; Accepted: 16 December 2021; Published online: 02 February 2022

\section{REFERENCES}

1. Cancer research institute. https://www.cancerresearch.org/scientists/immunooncology-landscape/fda-approval-timeline-of-active-immunotherapies.

2. Litchfield, K. et al. Meta-analysis of tumor- and T cell-intrinsic mechanisms of sensitization to checkpoint inhibition. Cell 184, 596-614.e14 (2021).

3. Aurisicchio, L., Pallocca, M., Ciliberto, G. \& Palombo, F. The perfect personalized cancer therapy: cancer vaccines against neoantigens. J. Exp. Clin. Cancer Res. 37, 86 (2018).

4. Castle, J. C. et al. Exploiting the mutanome for tumor vaccination. Cancer Res. 72, 1081-1091 (2012).

5. Yadav, M. et al. Predicting immunogenic tumour mutations by combining mass spectrometry and exome sequencing. Nature 515, 572-576 (2014).

6. Duan, F. et al. Genomic and bioinformatic profiling of mutational neoepitopes reveals new rules to predict anticancer immunogenicity. J. Exp. Med. 211, 2231-2248 (2014).

7. Schumacher, T. et al. A vaccine targeting mutant IDH1 induces antitumour immunity. Nature 512, 324-327 (2014).

8. Martin, S. D. et al. Low Mutation Burden in Ovarian Cancer May Limit the Utility of Neoantigen-Targeted Vaccines. 1-22 (2016) https://doi.org/10.1371/journal. pone.0155189.

9. Kuai, R., Ochyl, L. J., Bahjat, K. S., Schwendeman, A. \& Moon, J. J. Designer vaccine nanodiscs for personalized cancer immunotherapy. Nat. Mater. 16, 489-496 (2017).

10. Gubin, M. M. et al. Checkpoint blockade cancer immunotherapy targets tumourspecific mutant antigens. Nature 515, 577-581 (2014).

11. Zolkind, P. et al. Cancer immunogenomic approach to neoantigen discovery in a checkpoint blockade responsive murine model of oral cavity squamous cell carcinoma. 9, 4109-4119 (2018).

12. Kreiter, S. et al. Mutant MHC class II epitopes drive therapeutic immune responses to cancer. Nature 520, 692-696 (2015).

13. Kranz, L. M. et al. Systemic RNA delivery to dendritic cells exploits antiviral defence for cancer immunotherapy. Nature 534, 396-401 (2016).

14. Duperret, E. K. et al. A synthetic DNA, multi-neoantigen vaccine drives predominately MHC Class I CD8(+) T-cell responses, impacting tumor challenge. Cancer Immunol. Res. 7, 174-182 (2019).

15. Aurisicchio, L. et al. Poly-specific neoantigen-targeted cancer vaccines delay patient derived tumor growth. J Exp Clin Cancer Res. 4, 1-13 (2019).

16. Arbelaez, C. A. et al. OPEN A nanoparticle vaccine that targets neoantigen peptides to lymphoid tissues elicits robust antitumor $\mathrm{T}$ cell responses. npj Vaccines 1-14 https://doi.org/10.1038/s41541-020-00253-9.

17. Ott, P. A. et al. A phase lb trial of personalized neoantigen therapy plus Anti-PD-1 in patients with advanced melanoma, non-small cell lung cancer, or bladder cancer. Cell 183, 347-362.e24 (2020). 
18. Carreno, B. M. et al. Cancer immunotherapy. A dendritic cell vaccine increases the breadth and diversity of melanoma neoantigen-specific T cells. Sci. (80-.). 348, 803-808 (2015).

19. Ott, P. A. et al. An immunogenic personal neoantigen vaccine for patients with melanoma. Nature 547, 217-221 (2017).

20. Hilf, N. et al. Actively personalized vaccination trial for newly diagnosed glioblastoma. Nature 565, 240-245 (2019).

21. Fang, Y. et al. A pan-cancer clinical study of personalized neoantigen vaccine monotherapy in treating patients with various types of advanced solid tumors. Clin. Cancer Res. clincanres. 2881.2019 (2020). https://doi.org/10.1158/1078-0432. ccr-19-2881.

22. Biernacki, M. A. et al. CBFB-MYH11 fusion neoantigen enables T cell recognition and killing of acute myeloid leukemia. J. Clin. Invest. 130, 5127-5141 (2020).

23. Cafri, G. et al. mRNA vaccine-induced neoantigen-specific $T$ cell immunity in patients with gastrointestinal cancer. J. Clin. Invest. 130, 5976-5988 (2020).

24. Wei, S. C. et al. Distinct cellular mechanisms underlie anti-CTLA-4 and anti-PD-1 checkpoint blockade. Cell 170, 1120-1133.e17 (2017).

25. Boutros, C. et al. Safety profiles of anti-CTLA-4 and anti-PD-1 antibodies alone and in combination. Nat. Rev. Clin. Oncol. 13, 473-486 (2016).

26. Hailemichael, Y. et al. Cancer vaccine formulation dictates synergy with CTLA-4 and PD-L1 checkpoint blockade therapy. J. Clin. Invest. 128, 1338-1354 (2018).

27. Field, C. S. et al. Blocking CTLA-4 while priming with a whole cell vaccine reshapes the oligoclonal $\mathrm{T}$ cell infiltrate and eradicates tumors in an orthotopic glioma model. Oncoimmunology. 7, e1376154 (2018). https://doi.org/10.1080/ 2162402X.2017.1376154.

28. Lione, L. et al. Antitumor efficacy of a neoantigen cancer vaccine delivered by electroporation is influenced by microbiota composition. Oncoimmunology. 10, 1898832 (2021).

29. Castle, J. C. et al. Immunomic, genomic and transcriptomic characterization of CT26 colorectal carcinoma. BMC Genomics 15, 190 (2014).

30. Li, A. W., Sobral, M. C., Badrinath, S., Choi, Y. \& Graveline, A. A facile approach to enhance antigen response for personalized cancer vaccination. Nat. Mater. 17, 528-534 (2018)

31. D'Alise, A. M. et al. Adenoviral vaccine targeting multiple neoantigens as strategy to eradicate large tumors combined with checkpoint blockade. Nat. Commun. 10, 1-12 (2019).

32. Tondini, E. et al. A poly-neoantigen DNA vaccine synergizes with PD-1 blockade to induce T cell-mediated tumor control cell-mediated tumor control (2019). https://doi.org/10.1080/2162402X.2019.1652539.

33. Baharom, F. et al. Intravenous nanoparticle vaccination generates stem-like TCF1+ neoantigen-specific CD8+ T cells. Nat. Immunol. https://doi.org/10.1038/s41590020-00810-3 (2020).

34. Capietto, A.-H. et al. Mutation position is an important determinant for predicting cancer neoantigens. J. Exp. Med. 217, e20190179 (2020).

35. Swartz, A. M. et al. OPEN A conjoined universal helper epitope can unveil antitumor effects of a neoantigen vaccine targeting an MHC class 1-restricted neoepitope. npj Vaccines https://doi.org/10.1038/s41541-020-00273-5 (2021).

36. Salomon, N. et al. A liposomal RNA vaccine inducing neoantigen-specific CD4+ $T$ cells augments the antitumor activity of local radiotherapy in mice. Oncoimmunology 9 (2020).

37. Blass, E. \& Ott, P. A. Advances in the development of personalized neoantigenbased therapeutic cancer vaccines. Nat. Rev. Clin. Oncol. 18, 215-229 (2021).

38. Tondini, E. et al. A poly-neoantigen DNA vaccine synergizes with PD-1 blockade to induce T cell-mediated tumor control. Oncoimmunology. 8, 1652539 (2019).

39. Sharma, A. et al. Anti-CTLA-4 immunotherapy does not deplete FOXP3 p regulatory T cells (Tregs) in human cancers. 1233-1239 (2019) https://doi.org/ 10.1158/1078-0432.CCR-18-0762.

40. Hollern, D. P. et al. B cells and T follicular helper cells mediate response to checkpoint inhibitors in high mutation burden mouse models of breast cancer. Cell 179, 1191-1206.e21 (2019).

41. Bhojnagarwala, P. S., Perales-Puchalt, A., Cooch, N., Sardesai, N. Y. \& Weiner, D. B. A synDNA vaccine delivering neoAg collections controls heterogenous, multifocal murine lung and ovarian tumors via robust T cell generation. Mol. Ther. Oncolytics 21, 278-287 (2021).

42. Selby, M. J. et al. Preclinical development of ipilimumab and nivolumab combination immunotherapy: Mouse tumor models, In vitro functional studies, and cynomolgus macaque toxicology. PLoS One 11, 1-19 (2016).

43. Xu, C. et al. Efficient Lymph Node-Targeted Delivery of Personalized Cancer Vaccines with Reactive Oxygen Species-Inducing Reduced Graphene Oxide Nanosheets Efficient Lymph Node-Targeted Delivery of Personalized Cancer Vaccines with Reactive Oxygen Species-Inducing Red. (2020) https://doi.org/ 10.1021/acsnano.0c05062.

44. Kuai, R., Ochyl, L. J., Bahjat, K. S., Schwendeman, A. \& Moon, J. J. Designer vaccine nanodiscs for personalized cancer immunotherapy. Nat Mater. 16, $489-496$ (2016).
45. Krummel, B. M. F. \& Allison, J. R. CD28 and CTLA-4 have opposing effects on the response of T cells to stimulation. J Exp Med. 182, 459-465 (1995).

46. Ovcinnikovs, V. et al. CTLA-4-mediated transendocytosis of costimulatory molecules primarily targets migratory dendritic cells. Sci. Immunol. 4, eaaw0902 (2019).

47. Adam, L. et al. Innate molecular and cellular signature in the skin preceding longlasting T cell responses after electroporated DNA vaccination. J. Immunol. 204, 3375-3388 (2020).

48. Todorova, B. et al. Electroporation as a vaccine delivery system and a natural adjuvant to intradermal administration of plasmid DNA in macaques. 1-11 (2017) https://doi.org/10.1038/s41598-017-04547-2.

49. Verma, V. et al. PD-1 blockade in subprimed CD8 cells induces dysfunctional PD-1 +CD38hi cells and anti-PD-1 resistance. Nat. Immunol. 20, 1231-1243 (2019).

50. Philip, M. et al. Chromatin states define tumour-specific T cell dysfunction and reprogramming. Nature 545, 452-456 (2017).

51. Thibult, M. L. et al. Pd-1 is a novel regulator of human B-cell activation. Int. Immunol. 25, 129-137 (2013).

52. Wang, $X$. et al. PD-1-expressing B cells suppress CD4 + and CD8 + T cells via PD1/PD-L1-dependent pathway. Mol. Immunol. 109, 20-26 (2019).

53. Ahrends, T. et al. CD27 agonism plus PD-1 blockade recapitulates CD4+ T-cell help in therapeutic anticancer vaccination. Cancer Res. 76, 2921-2931 (2016).

54. Elia, L. et al. CD4+CD25+ regulatory T-cell-inactivation in combination with adenovirus vaccines enhances $\mathrm{T}$-cell responses and protects mice from tumor challenge. Cancer Gene Ther. 14, 201-210 (2007).

55. Lin, F. et al. Optimization of electroporation-enhanced intradermal delivery of DNA vaccine using a minimally invasive surface device. Hum. Gene Ther. Methods 23, 157-168 (2012).

\section{ACKNOWLEDGEMENTS}

We thank Eugenia Dogliotti for critical reading of the manuscript and Daniel Peluso for statistical assistance

\section{AUTHOR CONTRIBUTIONS}

F.P. and L.A. designed experiments while E.S., L.L., M.C., E.P., and A.C. carried out investigation and data analysis. F.P. wrote the original draft and with G.C. reviewed and edited the paper. All authors approved the final version.

\section{COMPETING INTERESTS}

The authors declare no competing interests.

\section{ADDITIONAL INFORMATION}

Supplementary information The online version contains supplementary material available at https://doi.org/10.1038/s41541-022-00433-9.

Correspondence and requests for materials should be addressed to Luigi Aurisicchio or Fabio Palombo.

Reprints and permission information is available at http://www.nature.com/ reprints

Publisher's note Springer Nature remains neutral with regard to jurisdictional claims in published maps and institutional affiliations.

Open Access This article is licensed under a Creative Commons Attribution 4.0 International License, which permits use, sharing, adaptation, distribution and reproduction in any medium or format, as long as you give appropriate credit to the original author(s) and the source, provide a link to the Creative Commons license, and indicate if changes were made. The images or other third party material in this article are included in the article's Creative Commons license, unless indicated otherwise in a credit line to the material. If material is not included in the article's Creative Commons license and your intended use is not permitted by statutory regulation or exceeds the permitted use, you will need to obtain permission directly from the copyright holder. To view a copy of this license, visit http://creativecommons. org/licenses/by/4.0/.

(c) The Author(s) 2022 